\title{
Clinical Pharmacology of the Unboosted HIV Integrase Strand Transfer Inhibitor (INSTI) Bictegravir (BIC)

\section{Introduction}

- Bictegravir (BIC; formerly GS-9883) is a novel,oncedaily, INSTI

- High barrier to resistance and potent in vitro activity against wild-type and most INSTI-resistant variants $^{1-4}$

- A 10 day study of BIC monotherapy in HIV-1 infected subjects demonstrated rapid decline in HIV-1 RNA >2 $\log _{10} 5$

- $\mathrm{BIC}$ single agent evaluated in Phase 2 in combination with emtricitabine (FTC) and tenofovir alafenamide $(\mathrm{TAF})^{6}$

- $\mathrm{BIC}$ is in Phase 3 clinical development as a single-tablet regimen (STR) coformulated with FTC and TAF for the treatment of HIV-1 infection

- An extensive Phase 1 program characterized the clinical pharmacology of BIC

\section{Results}

BIC Safety Profile from Phase 1 Program

- Generally well tolerated with no dose-dependen adverse events observed

- Evaluated BIC doses of 5 to $100 \mathrm{mg}$ in HIV-infected subjects and 5 to $600 \mathrm{mg}$ in healthy subjects

- No effect on QT interval based on a negative thorough QT study

- No impact on glomerular filtration as measured by ohexol clearance pharmacology of BIC

BIC Absorption, Distribution, Metabolism, Elimination (ADME)

Well absorbed $(>70 \%)$

- Highly bound to plasma proteins (>99\%)

- Primarily circulates as parent drug (BIC accounted for $68 \%$ plasma radioactivity)

- Metabolism is the major clearance pathway for BIC with similar contribution by oxidation (CYP3A4) and glucuronidation (UGT1A1)

Moderate hepatic impairment showed no clinically significant effect on PK

Minimal renal clearance $(\sim 1 \%$ of unchanged parent excreted in urine)

- No clinically significant effect of severe renal

impairment (eGFRCG $15-30 \mathrm{~mL} / \mathrm{min}$ ) on PK

BIC Pharmacokinetic ProfileHealthy Subjects
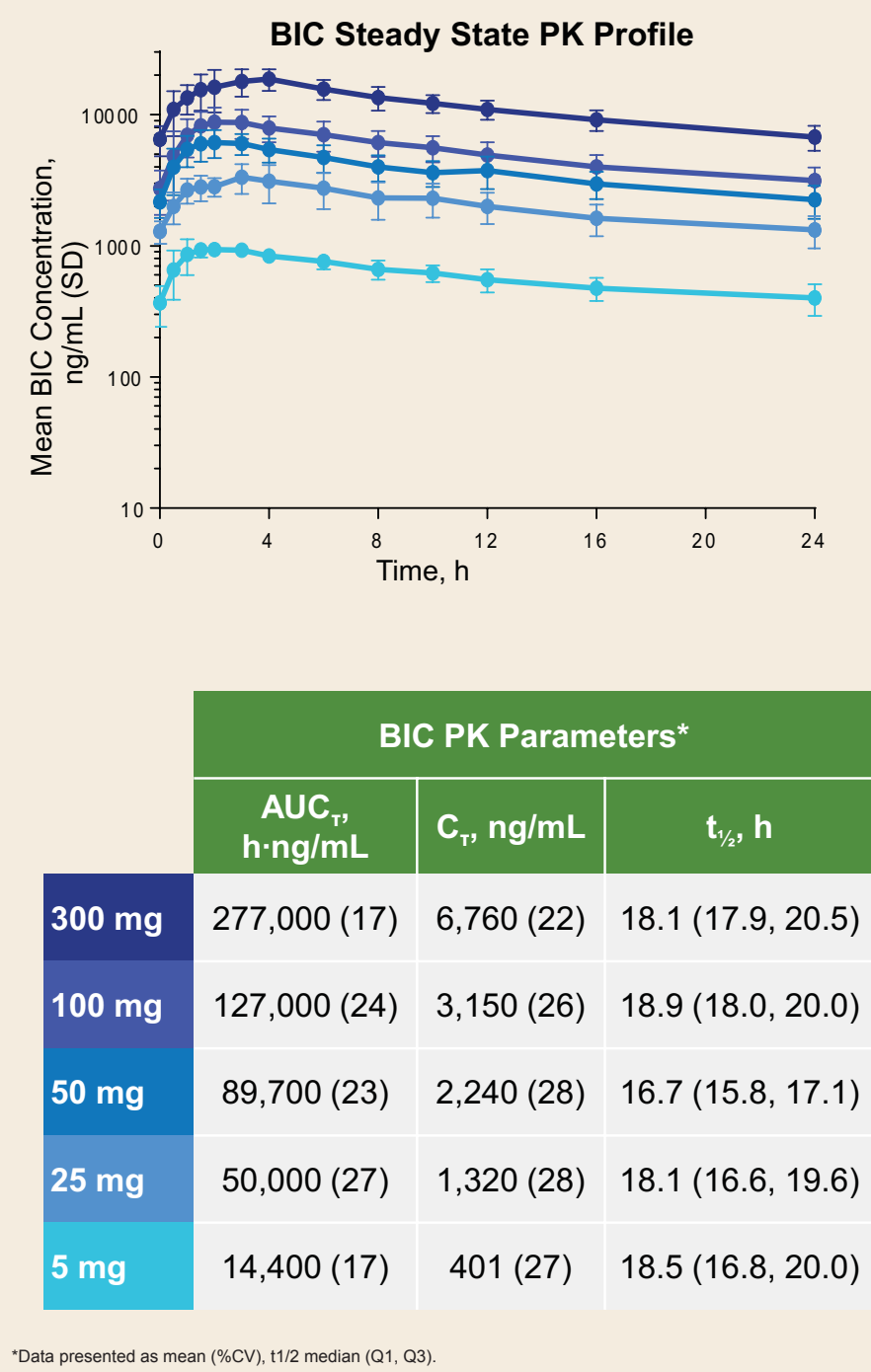

$t^{1} 12: \sim 18$ hours

PK profile supportive of once daily dosing

PK profile consistent with that observed in HIV-infected subjects'

\section{Results (Cont'd)}

BIC Pharmacokinetic Profile HIV-infected Subjects Phase 2: BIC 75 mg + F/TAF 200/25 mg
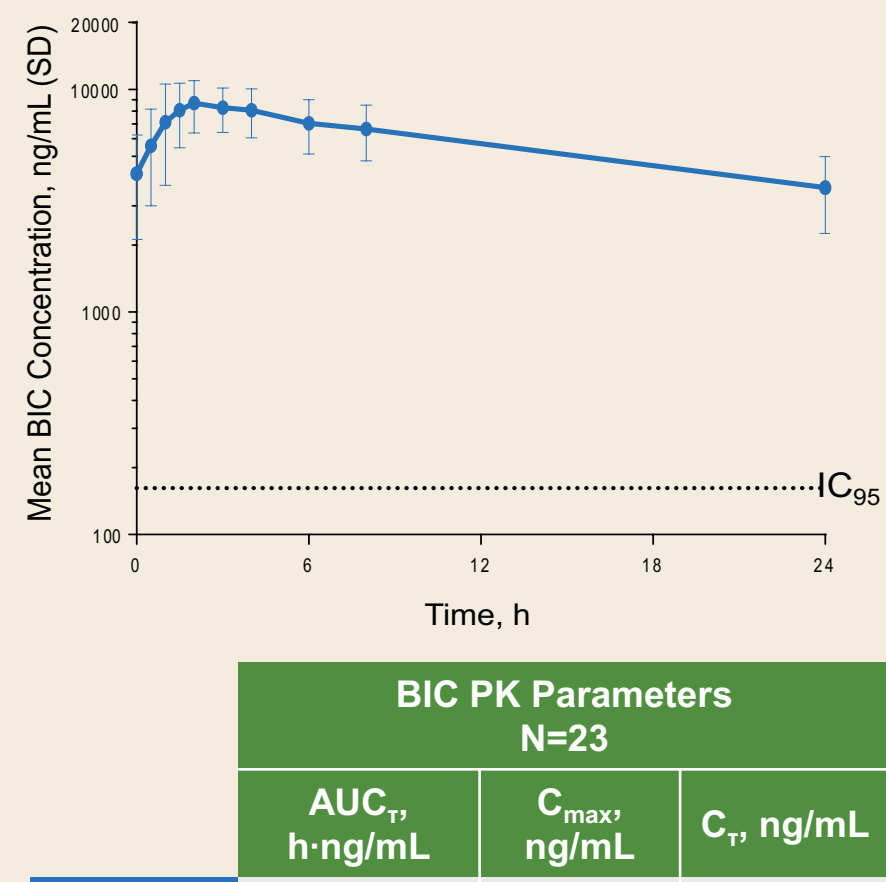

$\begin{array}{llll} & & \end{array} 5 \mathrm{mg} \quad 140,000(27) \quad 9340(27) \quad 3510(37)$

BIC Drug-Drug Interaction (DDI) Profile

Low potential as a victim of DDIs

- INSTIs are affected by cation-containing antacids BIC administration with antacids should be staggered ( 2 Fasted administration 2 hours before or 2 hours after antacid resulted in a decrease in BIC exposures of $13 \%$ and $52 \%$, respectively

BIC is a substrate of CYP3A4 and UGT1A Inibition of both CYP3A4 and UGT1A1 needed for

are to a clinically significan

BIC Drug-Drug Interaction ProfileClinical Study Probing Effect of Inhibitors or Inducers

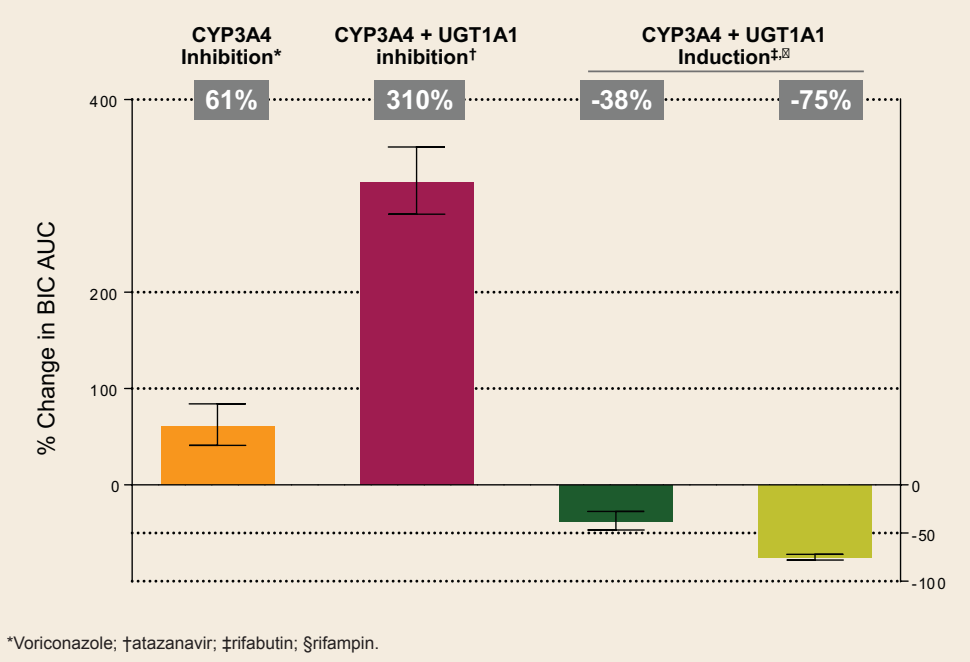

BIC Drug-Drug Interaction Profile

Effect of BIC on the PK of Coadministered Drugs

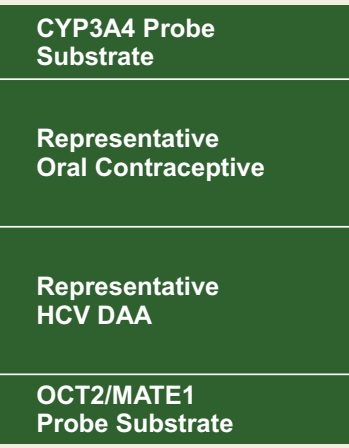

Midazolam

Norelgestromin EthinylEstradiol

Ledipasuir

Sofosbuvir

Metformin

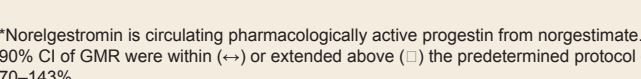

- Low potential to perpetrate DDIs Not an inhibitor or inducer of CYP3A4 or UGTA1 - No effect on midazolam

No interaction with a representative oral contraceptive - No effect on norgestimate/ethinyl estradiol

No interaction with a representative HCV DAA Limited liability for inhibition of renal transporters (OCT2 nd MATE
Coformulation of BIC + F/TAF into Single Tablet Regimen (STR)
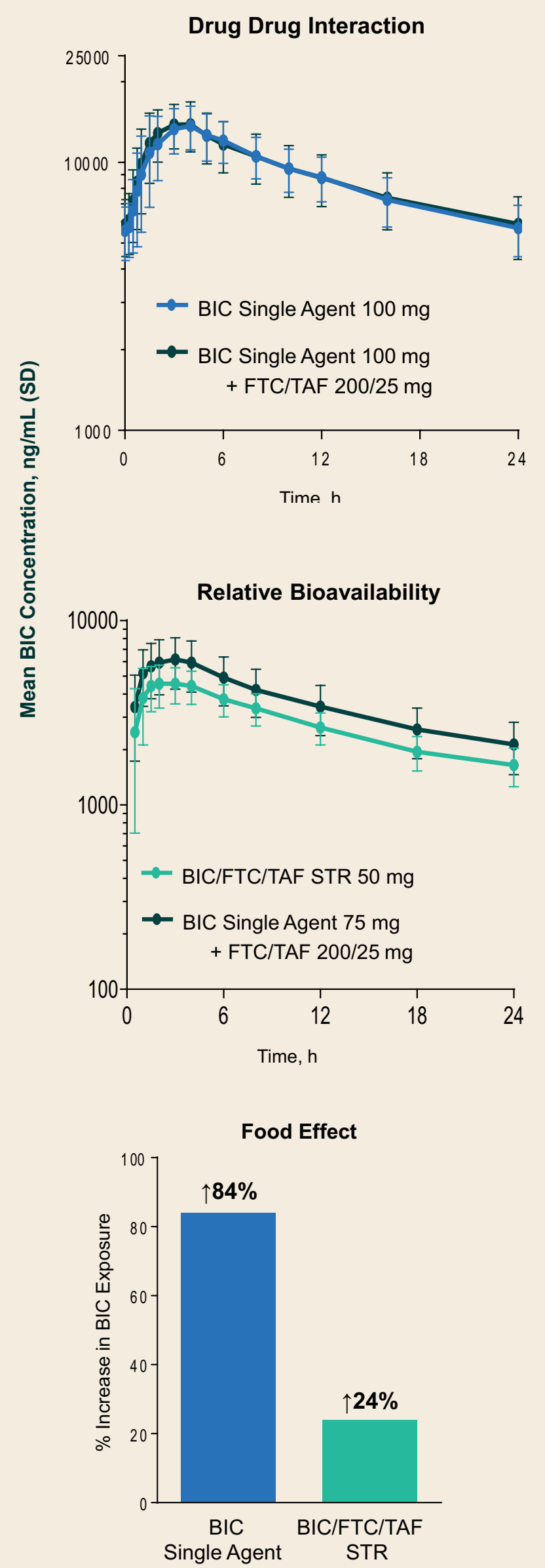

Lack of DDI between BIC and FTC/TAF established EFTC/TAF 200/25 mg dose

STR formulation development

Improved BIC bioavailability vs single agent Phase 2 formulation

Reduced food effect vs single agent Phase 2 formulation STR with $50 \mathrm{mg} \mathrm{BIC}$ dose selected for Phase 3:

\section{Conclusions}

- Bictegravir is an INSTI with pharmacokinetics supportive of once daily dosing and a favorable DDI profile

- Coformulated BIC/FTC/TAF 50/200/25 mg STR under evaluation in Phase 3 studies

\section{References}

Junes G, et al. ASM Microbe 2016, poster 1673;
. Lazerwith SE, et al. ASM Microbe 2016, poster 414

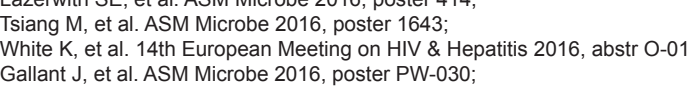

\section{Acknowledgements}

